\title{
Orientation and Velocity Dependence of the Nonequilibrium Partition Coefficient
}

K.M. Beatty, K.A. Jackson

Department of Materials Science and Engineering, The University of Arizona, Tucson AZ 85721

\begin{abstract}
Monte Carlo simulations based on a Spin-1 Ising Model for binary alloys have been used to investigate the non-equilibrium partition coefficient $\left(k_{n e q}\right)$ as a function of solid-liquid interface velocity and orientation. In simulations of $\mathrm{Si}$ with a second component $k_{n e q}$ is greater in the [111] direction than the [100] direction in agreement with experimental results reported by Aziz et al. The simulated partition coefficient scales with the square of the step velocity divided by the diffusion coefficient of the secondary component in the liquid.
\end{abstract}

\section{Introduction}

During rapid solidification of a binary alloy, the solid-liquid interface is not at equilibrium. The partition (or segregation) coefficient therefore differs from that determined from the equilibrium phase diagram. Rapid solidification leads to the development of novel microstructures and compositions not accessible by standard solidification techniques. Rapid solidification may be effected by such techniques as laser melting, splat cooling or melt atomization. $[1,2]$ Both analytical and computational models have been developed for $k_{n e q}$. The analytical model most extensively compared with experiment is due to Aziz.[3, 4] Jackson has investigated this phenomenon using a computational Spin-1 Ising model.[5, 6, 7] In addition, orientational dependence of the partition coefficient of $\mathrm{Bi}$ in $\mathrm{Si}$ during rapid solidification was experimentally investigated by Aziz et al. $[8]$

\section{Simulations of Solidification of Doped Silicon}

The probability of an atom going from a liquid to solid is given by: [9]

$$
P_{i}^{L S}=P_{o}\left(\delta_{a i}+\delta_{b i}\right) \exp \left(-\frac{\Delta S}{k_{B}}\right) \exp \left(-\sum_{j} \frac{\Phi_{i j}}{k_{B} T}\right)
$$


$\Phi_{i j}$ is the bond energy between an atom $i$ and an atom $j$. The energy of the bond between two atoms depends only on the types of atoms involved, and the relevant phase diagram. (In this paper the liquid was assumed to be an ideal solution, while the solid was assumed to be a regular solution.) The possible types of atoms are solid of species a, solid of species $b$, liquid of species $a$, and liquid of species $b$, which are represented by ' $A$ ' ' $B$ ' ' $a$ ', and 'b' respectively. $\Delta S$ is the entropy of fusion, $\delta_{t i}$ is one when atom $i$ is of type $t, k_{B}$ is Boltzmann's constant and $\mathrm{T}$ is the temperature. $P_{0}$ is a constant that will be determined if all the probabilities are to sum to one. Alternatively, if one considers the probabilities to be the likelihood of an event in a particular time interval, $P_{o}$ will determine the time scale. The probability of an atom going from crystal to liquid is given by:

$$
P_{i}^{S L}=P_{o}\left(\delta_{A i}+\delta_{B i}\right) \exp \left(-\sum_{j} \frac{\Phi_{i j}}{k_{B} T}\right)
$$

Diffusion is represented by an exchange between a liquid atom of type ' $b$ ' with a liquid atom of type ' $a$ ' with the following probability:

$$
P_{i}^{D}=P_{o} \Gamma \delta_{b i}\left(\sum_{j} \frac{\delta_{a j}}{Z}\right)
$$

Where $\Gamma$ is the diffusive jump frequency, $Z$ is the number of nearest neighbors. This will lead to a diffusion coefficient given by [10]

$$
D=\frac{\Gamma \alpha^{2}}{2 d}
$$

where $\alpha$ is the jump distance to the nearest neighbor and $d$ is the dimensionality of the lattice.

\section{Partition Coefficient Determination}

Under non-equilibrium conditions the gradient of concentration in the liquid near the interface can be very large, resulting in a concentration in the liquid that varies in atomic dimensions. Therefore care must be used in the definition of the segregation coefficient. A definition of $k_{n e q}$ can be based on the solution of the 1-d moving boundary problem:

$$
\frac{\partial u}{\partial t}=\frac{\partial}{\partial x}\left(D \frac{\partial u}{\partial x}-V u\right)
$$

where the concentration (represented by $u$ ) of the solid layer is $k_{\text {neq }}$ times the concentration of the neighboring liquid layer. The equation is differenced where $\Delta x$ does not approach zero, but is determined by the atomic lattice spacing. For silicon the (111) spacing of $3.13 \AA$ was chosen. The resulting difference equation is:

$$
u_{i}^{n+1}-u_{i}^{n}=\frac{\Delta t}{2 \Delta x} \sum_{s=n}^{n+1}\left(\frac{d_{l} u_{j-1}^{s}-\left(d_{l}+d_{r}\right) u_{j}^{s}+d_{r} u_{j+1}^{s}}{\Delta x}\right)+\left(b_{l} u_{j-1}^{s}-b_{m} u_{j}^{s}\right) V
$$




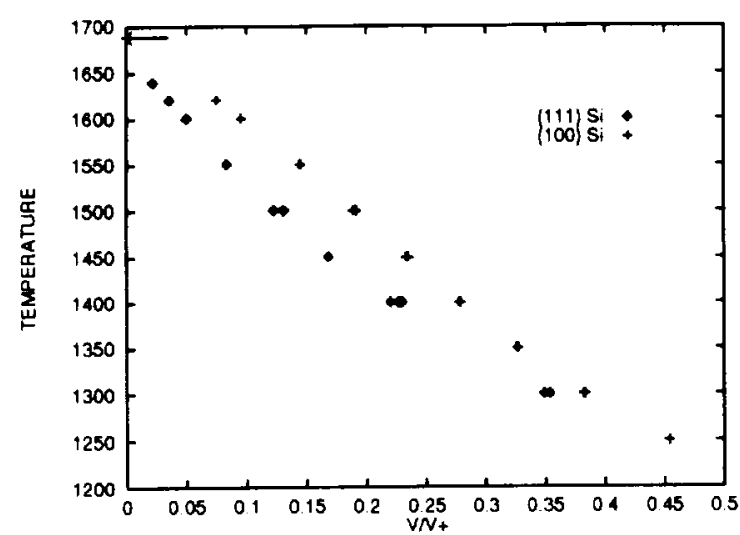

(a)

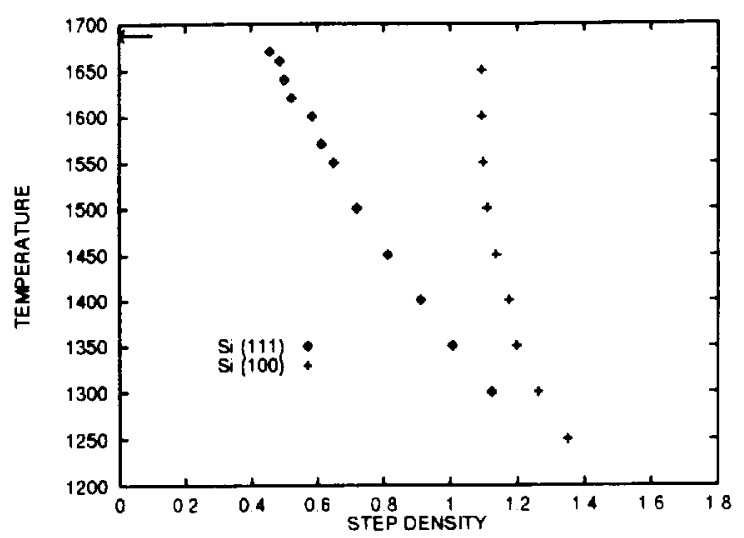

(b)

Figure 1: Ising model simulation results for silicon at different temperatures. (a) growth rate for the [100] and [111] growth directions. (b) Step density in the [111] and [100] directions.

The subscripts $1, m$, and $r$ refer to left middle and right, $i$ is the node number which is is increasing to the right, and $n$ is the time interval [11]. The fluxes can be considered to be into and out of individual atomic planes. The coefficients to use are given by Table 1 where B stands for the boundary layer, $L$ a liquid Layer, and $S$ the Solid Layer. B(L) represents the boundary with a liquid layer to its right, $(\mathrm{L}) \mathrm{L}$ represents a liquid layer with a liquid layer to its left.

TABLE 1

\begin{tabular}{|c|c|c|c|c|c|}
\hline & $d_{r}$ & $b_{m}$ & & $d_{l}$ & $b_{l}$ \\
\hline $\mathrm{B}(\mathrm{L})$ & 0 & 0 & $\mathrm{~B}$ & - & 0 \\
\hline $\mathrm{L}(\mathrm{L})$ & $\mathrm{D}$ & 1 & $(\mathrm{~B}) \mathrm{L}$ & 0 & 1 \\
\hline $\mathrm{L}(\mathrm{S})$ & 0 & $\mathrm{k}$ & $(\mathrm{L}) \mathrm{L}$ & $\mathrm{D}$ & 1 \\
\hline $\mathrm{S}$ & - & 1 & $(\mathrm{~L}) \mathrm{S}$ & 0 & $\mathrm{k}$ \\
\hline
\end{tabular}

Using this procedure a non-equilibrium partition coefficient can be fit to the Monte Carlo simulation results ( using the appropriate diffusion coefficient and growth velocity). Figure 2 shows a comparison of finite difference calculations and Monte Carlo simulations.

\section{Scaling of Monte Carlo Simulation Parameters}

The paramaters in the Spin-1 Ising Model are the nearest neighbor bond energies, the latent heat of fusion at the melting temperature, the diffusive jump frequency $\Gamma$, and the crystal structure. For the present work a diamond cubic structure with a lattice spacing of $5.43 \AA$ was used. In order for the Ising model to give the proper surface roughening temperatures, an entropy of fusion greater than the experimental value must be used. The value for $\Delta S / k_{B}$ used in the simulations was 6 . Interpretation of the results depends 


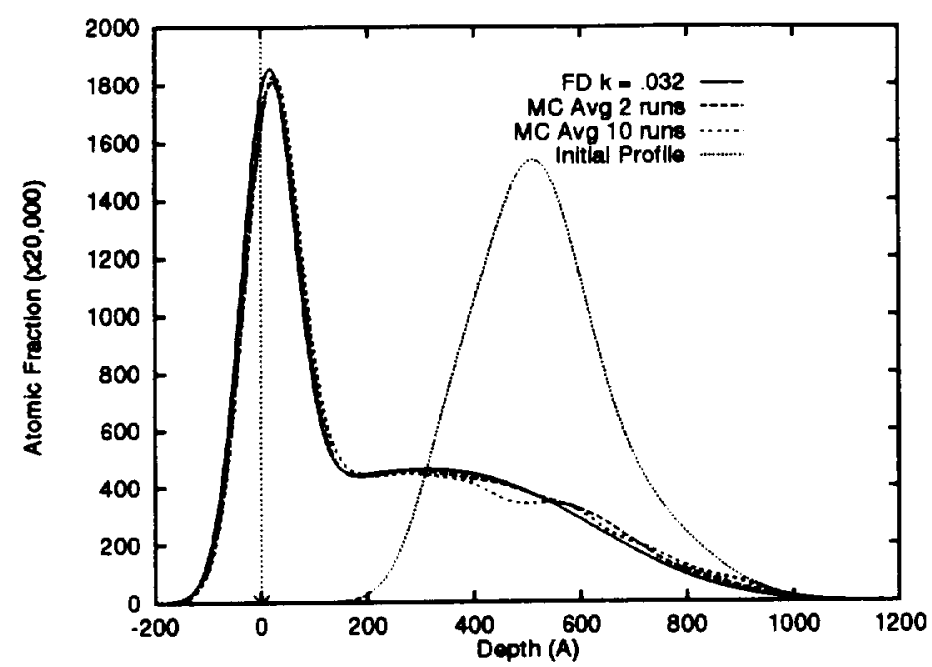

Figure 2: The initial concentration profile was the same for all simulations. Concentration profiles of the solid were all convoluted with a Gaussian before comparison (convoluted profiles shown). Both series of $\mathrm{MC}$ simulations were in the [111] direction with $\Omega=10$ and a temperature of $1450 \mathrm{~K}$. The parameters for the finite difference run were $D=2 \times 10^{-4} \mathrm{~cm}^{2} / \mathrm{s}$, a velocity of $14.4 \mathrm{~m} / \mathrm{s}, k_{n e q}$ value of 0.32 , and a grid spacing of $3.13 \AA$. In this case only the value of $k_{n e q}$ was used as a free parameter for fitting the finite difference run to the Ising model simulation results.

on $P_{o}$ in equations 1,2 and 3. By matching the growth velocity in the -simulations to experimental growth velocities it was found the time scale could be changed to seconds by setting $P_{o} \approx 9 \times 10^{13}$. (A single value for $P_{o}$ was found by fitting experimental data at $1550 \mathrm{~K}$. In general $P_{0}$ should account for a temperature dependent arrival rate at steps.) $\Gamma$ is defined to be:

$$
\Gamma=\Omega R_{a}
$$

where $R_{a}$ is the arrival rate at a step, and $\Omega$ is a parameter which can be adjusted to match diffusion data. For data chosen equation 1 simplifies to:

$$
R_{a}=P_{0} \exp \left(-\Delta S / k_{B}\right)
$$

Using equation 4 for diamond cubic and a value of 10 for $\Omega$, which is reasonable on the basis of atomic considerations [12], we have:

$$
D=\frac{\Omega R_{a} \alpha^{2}}{6}=\frac{\Omega R_{a} a_{o}^{2}}{32} \approx 2 \times 10^{-4} \mathrm{~cm}^{2} / \mathrm{s}
$$

\section{Results and Discussion}

Figure 1 (a) shows simulation results for growth of silicon in the [111] and [100] directions. The line for the [111] direction should come in vertically to the melting point of silicon since the surface is smooth. Figure 1 (b) shows the step density as a function of undercooling 


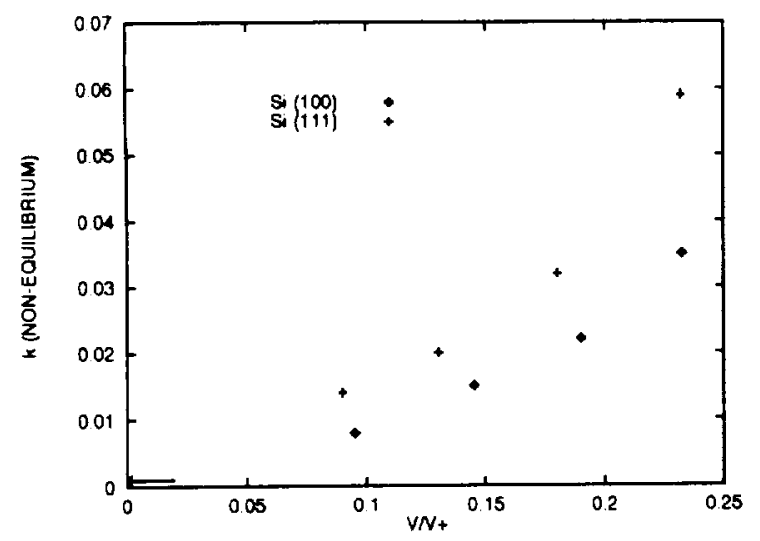

(a)

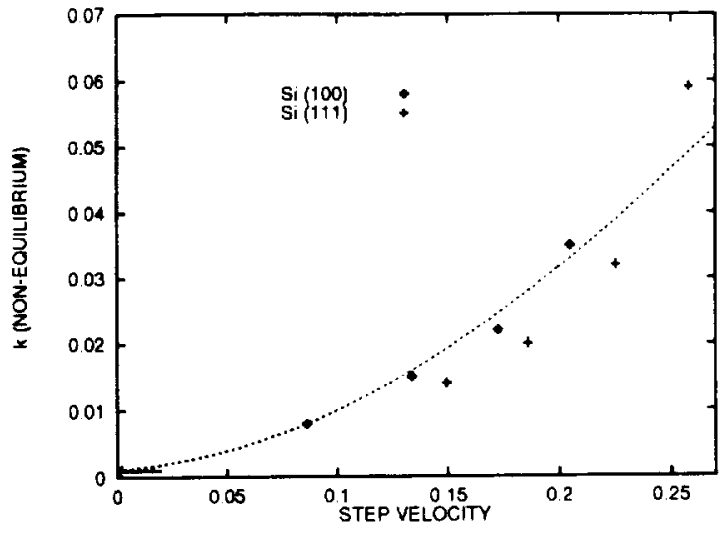

(b)

Figure 3: Ising model simulations for the diamond cubic structure in the [111] and [100] directions with $\Omega=10$. A horizontal line is drawn at $k_{e q}=.001$. (a) $k_{n e q}$ as a function of normalized velocity. (b) $k_{n e q}$ as a function of normalized step velocity.

for the (111) and (100) faces. The step density is defined to be the number of liquid atoms at the interface with at least half their bonds to solid neighbors divided by the cross-sectional area of the interface. Figure 2 is an example fitting of a finite difference run to Monte Carlo simulations. Spurious peaks found in the average of two Ising model simulations is not found in the average of ten runs. Figure 3 gives results for $k_{n e q}$ as a function of normalized growth velocity in the [111] and [100] directions. At $7 \mathrm{~m} / \mathrm{s}$ the Ising model simulations result in a factor of approximately 2 . Aziz et al. found that this ratio was about 5 at $1.7 \mathrm{~m} / \mathrm{s}$ and about 3 at $5 \mathrm{~m} / \mathrm{s}[8]$. The ratio $k_{n e q(111)} / k_{\text {neq }(100)}$ found by experiment and simulation are in reasonable agreement. Figure $3(b)$ plots the data for both $k_{n e q(111)}$ and $k_{n e q(100)}$ versus the normalized step velocity. (The normalized step velocity is the normalized growth velocity divided by the density of steps.) The results for both growth directions fall on a single curve when $k_{n e q}$ is plotted as a function of step velocity.

\section{Conclusions}

The Monte Carlo simulations suggest that the determining factor in solute trapping is the step velocity at the interface. The Spin-1 Ising model predicts greater incorporation of an impurity in $\mathrm{Si}$ with $k_{e q}=.001$ on the (111) face than the (100) face for the same growth velocity. The magnitude of the effect is in reasonable agreement with experimental results.

\section{Acknowledgement}

This work was supported by NASA Contract Number NAG8-944. 


\section{References}

[1] P. Duwez, R.H. Willens, and W. Klement Jr. Applicd Physics 31, 1136 (1960).

[2] D. Beck, S.M. Copley, and M. Bass. Metall. Trans. A 13, 1879 (1982).

[3] M.J. Aziz, J.Y. Tsao, M.O. Thompson, P.S. Peercy, and C.W. White. Phys. Rfv. Lett. 56, 2489 (1986).

[4] M.J. Aziz and T. Kaplan. Acta Metall. 36, 2335 (1988).

[5] K.A. Jackson, G.H. Gilmer, D.E. Temkin, J.D. Weinberg, and K. Beatty. Journal of Crystal Growth 128(1-4), 127-138 (1993).

[6] K.A. Jackson, G.H. Gilmer, and D.E. Temkin. Phys. Rev. Lett. 75(13), 25.30 (1995).

[7] K.A. Jackson, G.H. Gilmer, D.E. Temkin, and K.M. Beatty. Journal of Crystal Growth to be published (1995).

[8] M.J. Aziz and (..W. White. Phys. Rev. Lett. 57(21), 2675 (1986).

[9] G.H. Gilmer. Mater. Sci. Eng. 65, 15 (1984).

[10] P. Shewmon. Diffusion in Solids and ed. Minerals Metals and Materials Society (1989).

[11] W.H. Press, S.A. Teukolsky, W.T. Vetterling, and B.P. Flannery. Numerical Recipes in C 2nd ed. Cambridge University Press (1992).

[12] M.H. Grabow, G.H. Gilmer, and A.F. Bakker. MRS Symposium Proceedings 141, 349 (1989). 